\title{
Activation of peroxisome proliferator-activated receptor $\gamma$ inhibits vascular calcification by upregulating Klotho
}

\author{
LIJUAN CHENG ${ }^{1}$, LEI ZHANG ${ }^{1}, \mathrm{JUN} \mathrm{YANG}^{2}$ and $\mathrm{LIRONG} \mathrm{HAO}{ }^{1}$ \\ ${ }^{1}$ Department of Nephrology, The First Affiliated Hospital of Harbin Medical University, Harbin, Heilongjiang 150001; \\ ${ }^{2}$ Department of Nephrology, Daqing Oilfield General Hospital, Daqing, Heilongjiang 163001, P.R. China
}

Received July 22, 2015; Accepted October 24, 2016

DOI: $10.3892 /$ etm.2016.3996

\begin{abstract}
Cardiovascular diseases are common in patients with chronic kidney disease. One of the key symptoms is the calcification of the vascular smooth muscle cells (VSMCs), which is induced by dysregulated mineral metabolism with high circulating levels of inorganic phosphate $(\mathrm{Pi})$ and calcium. Klotho, which was originally identified as an aging suppressor gene, has been shown to be associated with vascular calcification. Since Klotho was recently identified as a target for nuclear receptor peroxisome proliferator-activated receptor (PPAR) $\gamma$, the present study aimed to determine whether PPAR $\gamma$ regulates VSMC calcification through modulating the expression levels of Klotho. It was demonstrated that the expression of PPAR $\gamma$ was downregulated during Pi-induced VSMC calcification. In addition, treatment with PPAR $\gamma$ agonists inhibited the calcification and enhanced the expression of Klotho in VSMCs in a PPAR $\gamma$-dependent manner. Of note, loss of Klotho expression by RNA interference abolished the ability of PPAR $\gamma$ activation to inhibit VSMC calcification. Furthermore, activation of Klotho as well as PPAR $\gamma$ inhibited the expression of Pi transporter 1/2 and reduced Pi influx into VSMCs. To the best of our knowledge, the present study was the first to demonstrate that PPAR $\gamma$ regulates VSMC calcification through activating Klotho.
\end{abstract}

\section{Introduction}

Cardiovascular diseases are the leading cause of mortality in patients with chronic kidney disease (CKD) (1). Studies have demonstrated that declining renal function in CKD is associated with an elevated risk of cardiovascular disease $(2,3)$. One of the key factors leading to the increased burden of

Correspondence to: Dr Lirong Hao, Department of Nephrology, The First Affiliated Hospital of Harbin Medical University, 23 Youzheng Street, Harbin, Heilongjiang 150001, P.R. China E-mail: stevenbee@163.com

Key words: vascular smooth muscle cell calcification, peroxisome proliferator-activated receptor $\gamma$, Klotho, inorganic phosphate transporter $1 / 2$ cardiovascular disease is the calcification of the vascular smooth muscle cells (VSMCs) lining the vessel wall, and the severity and histoanatomic type of vascular calcification are predictors of subsequent vascular mortality (4). The prevalence and progression of vascular calcification are markedly increased in patients with advanced CKD (5). Vascular calcification in CKD patients is known as medial artery calcification, which is distinct from that found in patients with atherosclerosis, and is characterized by amorphous minerals forming along one or more elastic lamellae of the medial layer $(6,7)$. For a long period, vascular calcification was thought to be a passive process resulting from elevated phosphate $(\mathrm{Pi})$ levels and increased calcium Pi products in the plasma (8-10). However, other studies have established that vascular calcification in CKD is a highly regulated cell-mediated process that involves the entry of VSMCs into a transdifferentiation program of osteogenesis, during which numerous key regulators of bone formation and bone structural proteins are expressed (11-14).

Despite these findings, dysregulated mineral metabolism with high circulating levels of $\mathrm{Pi}$ and calcium have been demonstrated to be the most important factors for the initiation and progression of the calcification process in CKD patients $(1,15)$. Extracellular Pi promotes VSMC mineralization in a concentration- and time-dependent manner by increasing Pi influx via the sodium-dependent Pi co-transporters, Pi transporter (piT)-1 and PiT-2, leading to the induction of osteoblastic differentiation factors, including core-binding factor alpha 1 (Cbfa1)/runt-related transcription factor 2 (Runx2) (16). Either the blockade of PiT-1/2 or inhibition of PiT-1/2 activity by small interfering (si)RNA prevents the induction of Cbfa1/ Runx2 and osteoclast expression in VSMCs even under high extracellular Pi concentrations (17), suggesting that elevated extracellular Pi concentrations induce the mineralization of VSMCs through the activation of PiT-1/2. In addition to extracellular $\mathrm{Pi}$, calcium accelerates the mineralization of VSMCs $(18,19)$. In addition, calcium-induced mineralization has been demonstrated to be also dependent on the function of PiT-1/2 (19).

A growing body of evidence has demonstrated that the function of Klotho is associated with vascular calcification. The Klotho gene was originally identified as an aging suppressor gene in mice (20). It encodes a single span transmembrane protein and is expressed primarily in renal tubular epithelial cells (20). Studies have indicated that 
Klotho deficiency promotes calcification and osteoblastic differentiation of VSMCs $(21,22)$, whereas Klotho transgenic mice have better preserved renal function and markedly less calcification compared with wild-type mice with CKD (21). It has been suggested that Klotho suppresses osteoblastic transdifferentiation and calcification of VSMCs by inhibiting PiT-1/2-dependent Pi uptake, thus repressing the expression of Cbfa1/Runx2 (21). Klotho has been identified as a target for nuclear receptor peroxisome proliferator-activated receptor (PPAR) $\gamma$ (23). Thiazolidinediones, which act as PPAR $\gamma$ agonists, increase Klotho expression in HEK293 cells several renal epithelial cell lines at the mRNA and protein level. This induction was blocked by siRNA-mediated gene silencing of PPAR $\gamma$ or PPAR $\gamma$ antagonists, which have been shown to attenuate high glucose-induced VSMC calcification (24). However, the underlying mechanisms of the increased expression of Klotho have remained elusive.

The present study demonstrated that the expression of PPAR $\gamma$ was downregulated during Pi-induced VSMC calcification. In addition, treatment with PPAR $\gamma$ agonists was found to inhibit the calcification and enhanced the expression of Klotho in VSMCs in a PPAR $\gamma$-dependent manner. Of note, loss of Klotho expression by RNA interference abolished the ability of PPAR $\gamma$ activation to inhibit VSMC calcification. To the best of our knowledge, the present study was the first to demonstrate that PPAR $\gamma$ regulates VSMC calcification through activating Klotho.

\section{Materials and methods}

Cell culture. Vascular smooth muscle cells (VSMCs) were obtained by a previously described explant method $(25,26)$. In brief, tissue was separated from simmental bovine aorta segments (Harbin Bin Good Animal Husbandry Co., Ltd., Harbin, China). Small pieces of tissue $\left(1-2 \mathrm{~mm}^{3}\right)$ were placed into a $10-\mathrm{cm}$ culture dish and cultured for several weeks in Dulbecco's modified Eagle's medium (DMEM) containing $4.5 \mathrm{~g} / 1$ glucose (Invitrogen; Thermo Fisher Scientific, Inc., Waltham, MA, USA) supplemented with $15 \%$ fetal bovine serum and $10 \mathrm{mM}$ sodium pyruvate (both Invitrogen; Thermo Fisher Scientific, Inc.) in a humidified atmosphere containing $5 \% \mathrm{CO}_{2}$ at $37^{\circ} \mathrm{C}$. Cells that had migrated from the explants were collected and maintained in DMEM containing $1.0 \mathrm{~g} / \mathrm{l}$ glucose (Invitrogen; Thermo Fisher Scientific, Inc.), 15\% FBS supplemented with $10 \mathrm{mM}$ sodium pyruvate. For calcification experiments, cells of up to passage 8 were used.

Induction of calcification. VSMCs were seeded on 24-well plates at a density of $5 \times 10^{4} /$ well. At confluence, cells were incubated in calcification medium (DMEM containing 15\% FBS and $1 \mathrm{mM}$ sodium pyruvate in the presence of $3 \mathrm{mM}$ inorganic P (Pi; Invitrogen; Thermo Fisher Scientific, Inc.) for up to 9 days. Every 2 days, the medium was replaced with fresh medium. For time-course experiments, the first day of culture in calcification medium was defined as day 0 . Cells incubated in calcification medium with $1 \mathrm{mM}$ Pi were used as controls.

Activation of PPAR $\gamma$. VSMCs were administered with various concentrations $(0,4,8,12,16$ or $20 \mu \mathrm{M})$ of the PPAR $\gamma$ agonists rosiglitazone (RGZ; Santa Cruz Biotechnology, Inc.,
Dallas, TX, USA) and thiazolidinedione (TZL; Santa Cruz Biotechnology, Inc.) in the presence of $3 \mathrm{mM}$ Pi for 9 days. VSMCs were subsequently administered with RGZ or TZL in the absence or presence of PPAR $\gamma$ inhibitor GW9662 $(10 \mu \mathrm{M}$; Santa Cruz Biotechnology, Inc.) to confirm the role of PPAR $\gamma$ in Pi-induced calcification.

Quantification of calcium deposition. Cells were decalcified with $0.6 \mathrm{~N} \mathrm{HCl}$ for $24 \mathrm{~h}$. Subsequently, the cells were solubilized with $0.1 \mathrm{~N} \mathrm{NaOH} / 0.1 \%$ SDS. The calcium content was determined colorimetrically by the o-cresolphthalein complexone method (Calcium kit; Nanjing Jiancheng Bioengineering Institute, Nanjing, China). The protein content was measured with a bicinchoninic acid (BCA) protein assay kit (Thermo Fisher Scientific, Inc.). The calcium content was normalized to protein content.

Von Kossa staining. Cells were fixed with $0.1 \%$ glutaraldehyde in Pi-buffered saline for $15 \mathrm{~min}$ at room temperature, and were then washed 3 times with double distilled $\mathrm{H}_{2} \mathrm{O}$. Cells were incubated with $5 \%$ silver nitrate solution and exposed to sunlight for $30 \mathrm{~min}$, washed with distilled water for $5 \mathrm{~min}$ and treated with 5\% sodium thiosulfate for $2 \mathrm{~min}$. Images of calcium particles were captured at 40x magnification (C-5060; Olympus Corp., Tokyo, Japan) under a microscope (TH4-200; Olympus Corp.).

Alkaline phosphatase staining and activity assay. Alkaline phosphatase (ALP) staining and activity assay were performed using a 5-bromo-4-chloro-3-indolyl Pi/nitro-blue tetrazolium Alkaline Phosphatase Staining kit and an Alkaline Phosphatase Assay kit (Beyotime Institute of Biotechnology, Shanghai, China), respectively, according to the manufacturer's protocol. Images of samples stained for ALP were captured at 40x magnification (C-5060; Olympus Corp.) under a microscope (TH4-200; Olympus Corp.). ALP activity was normalized to the total protein concentration determined using the BCA protein assay (Thermo Fisher Scientific, Inc.).

Reverse-transcription quantitative polymerase chain reaction (RT-qPCR) analysis. Total RNA from VSMCs was isolated using TRIzol reagent (Invitrogen). Complementary DNA was generated from $2 \mu \mathrm{g}$ RNA with Oligo-dT primers by using the SuperScript III First Stand Synthesis System (Thermo Fisher Scientific, Inc.) according to the manufacturer's protocol. Real-time PCR was performed in a $20-\mu 1$ reaction mixture containing $1 \mu \mathrm{l}$ cDNA, $9 \mu \mathrm{l}$ water and $10 \mu \mathrm{l}$ 2X TaqMan PreAmp Master Mix (Invitrogen). Amplification was performed in an ABI 7300 system (Applied Biosystems; Thermo Fisher Scientific, Inc.) with Taqman green fluorescence (Invitrogen). The forward and reverse PCR primers, and their respective probes were as follows: PPAR $\gamma$ (forward: 5'-CATAATGCCATCAGGTTTGG-3'; reverse: 5'-GTCAGC AGACTCTGGGTTCA-3'; probe: 5'-CTTCTCGGCCTG TGGCATGC-3'), Klotho (forward: 5'-GGTGGATGTCAT TGGGTACA-3'; reverse: 5'-AGAGTCCACGTCTGATGC TG-3'; probe: 5'-CGGTGCCACTCGAAGCCATC-3'), Runx2 (forward: 5'-ATGGTTAATCTCCGCAGGTC-3'; reverse: 5'-TGGTGTCACTGTGCTGAAGA-3'; probe: 5'-CCAGCC ACCGAGACCAACAGA-3'), Msh homeobox 2 (Msx2) 
(forward: CCAGCTCTCTGAACCTCACA-3'; reverse: 5'-AGTTCTGCCTCCTGCAGTCT-3'; probe: 5'-CGCCTT GGCTCTTCGGTTCTG-3'), osteocalcin (OCN) (forward: 5'-GAGCTCAACCCTGACTGTGA-3'; reverse: 5'-CTAGAC TGGGCCGTAGAAGC-3'; probe: 5'-CCACATCGGCTT CCAGGAAGC-3'), matrix gla protein (MGP) (forward: 5'-GAGAACTCAACAAGCCTCA-3'; reverse: 5'-TCGTAG GCAGCATTGTATCC-3'; probe: 5'-CAAGCTTCCCGGTTG AGCTCG-3'), SM22 $\alpha$ (forward: 5'-AGCAAGCTGGTCAAT AGCCT-3'; reverse: 5'-CTCCATCTGCTTGAAGACCA-3'; probe: 5'-CGGGCACCTTCACTGGCTTG-3') and $\alpha$-actin (forward: 5'-GGTGACTCTCCAAGGTGGAT-3'; reverse: 5'-TGCAAAGGCTGAACAAACTC-3'; probe: 5'-CCTTGG CTGGGCATCACCCT-3'), PiT1 (forward: 5'-GATGTC CACAGCAACAGGAC-3'; reverse: 5'-ACTCAACGTGTG GTCAGGAA-3'; probe: 5'-AAGCTCCCTGCCATCACG CC-3'), PiT2 (forward: 5'-GGGAGGGTTGACCTGTTA GA-3'; reverse: 5'-AGTCGTACTTCCTCCCGCTA-3'; probe: 5'-AGCAACCAGGGATGCTCCGC-3'). $\beta$-actin (forward: 5'-AGCAGATGTGGATCAGCAAG-3'; reverse: 5'-TAACAG TCCGCCTAGAAGCA-3'; probe: 5'-CCTCCATCGTCCACC GCAAA-3'). All primers were obtained from Thermo Fisher Scientific, Inc., and the following thermocycling conditions were used: $95^{\circ} \mathrm{C}$ for $8 \mathrm{~min}, 40$ cycles of $95^{\circ} \mathrm{C}$ for $20 \mathrm{sec}$, and $60^{\circ} \mathrm{C}$ for $1 \mathrm{~min}$. The relative expression levels of mRNA were calculated using the $2^{-\Delta \Delta \mathrm{Ct}}$ methods as described previously (27).

Western blot analysis. Cells were lysed in radioimmunoprecipitation buffer supplemented with a protease and phosphatase inhibitor cocktail (Thermo Fisher Scientific, Inc.). The protein concentration was determined using the BCA protein assay. Proteins were subjected to SDS-polyacrylamide gel electrophoresis and subsequently transferred onto a nitrocellulose membrane. Following blocking with $3 \%$ bovine serum albumin, the membrane was incubated with primary antibodies, including anti-PPAR $\gamma$ (1:500; ab66343; Abcam, Cambridge, UK), anti-Klotho $(1: 1,000 ;$ ab203576; Abcam) and anti- $\beta$-actin (1:500; ab1801; Abcam) overnight at $4{ }^{\circ} \mathrm{C}$. Subsequently, membranes were incubated with horseradish peroxidase-conjugated secondary antibody (1:10,000; BM2006; Wuhan Boster Biological Technology, Ltd., Wuhan, China) for $1 \mathrm{~h}$ at room temperature. Protein bands were detected using an enhanced chemiluminescence western blotting detection kit (Thermo Fisher Scientific, Inc.), followed by exposure of the membranes to X-ray film. Image J software (version 1.140; US National Institutes of Health, Bethesda, MD, USA) was used to quantify the blot intensity.

Small interfering RNA (siRNA) and transfection. PPAR $\gamma$ siRNA, Klotho siRNA and control scrambled siRNA (Invitrogen; Thermo Fisher Scientific, Inc.) were transfected with Lipofectamine RNAiMAX (Invitrogen; Thermo Fisher Scientific, Inc.) according to the manufacturer's instructions. Cells were seeded in 24-well plates at $5 \times 10^{4} /$ well and were grown overnight to reach $\sim 80 \%$ confluence. The cells were transfected with 30 pmol siRNA and incubated for $48 \mathrm{~h}$, and used for subsequent experiments after the transfection efficiency was confirmed by western blot analysis.
Statistical analysis. Values are expressed as the mean \pm standard error of the mean. Differences among groups were analyzed by analysis of variance followed by Bonferroni post-hoc analyses as appropriate. $\mathrm{P}<0.05$ was considered to indicate a statistically significant difference. All statistical analyses were performed using SPSS 18.0 Version software (SPSS, Inc., Chicago, IL, USA).

\section{Results}

PPAR expression is downregulated in VSMCs with Pi-induced calcification. In order to investigate the role of PPAR $\gamma$ activation in VSMC calcification, the expression levels of PPAR $\gamma$ during Pi-induced VSMC calcification were examined. While low ambient $\mathrm{Pi}(1 \mathrm{mM})$ had little effect on calcium deposition, high ambient Pi (3 mM) markedly increased calcium deposition in VSMCs in a time-dependent manner. The calcium content in these cells was elevated from $21 \pm 3.4 \mu \mathrm{g} / \mathrm{ml}$ on day 0 to $386 \pm 31.7 \mu \mathrm{g} / \mathrm{ml}$ on day 9 (Fig. 1A). During Pi-induced calcification in VSMCs, the mRNA (Fig. 1B) and the protein levels (Fig. 1C) of PPAR $\gamma$ were significantly downregulated.

PPAR agonists inhibit Pi-induced VSMC calcification. To examine whether PPAR $\gamma$ activation can suppress the calcification of VSMCs, the cells were treated with various concentrations of the PPAR $\gamma$ agonists RGZ and TZL in the presence of $3 \mathrm{mM}$ Pi for 9 days. The results showed that RGZ and TZL decreased Pi-induced calcium deposition in VSMCs in a dose-dependent manner, with a mean effective dose of $7.14 \mu \mathrm{M}$ for RGZ and $8.98 \mu \mathrm{M}$ for TZL (Fig. 2A). Consistently, Von Kossa staining (28), ALP staining (29) (Fig. 2B) and the ALP activity assay (Fig. 2C) also revealed that Pi-induced calcification in VSMCs was inhibited by RGZ and TZL.

To further investigate whether PPAR $\gamma$ agonists modulate VSMC transdifferentiation, the mRNA levels of osteogenic genes and smooth muscle lineage markers were examined in VSMCs by RT-qPCR. While Pi significantly increased the expression of the osteogenic differentiation marker genes Runx2, Msx2 and OCN, the expression of these osteogenic genes was suppressed by RGZ and TZL (Fig. 2D). Conversely, the mRNA levels of calcification inhibitor gene MGP and smooth muscle differentiation marker genes SM22 $\alpha$ and $\alpha$-actin were significantly reduced during Pi-induced calcification, but were upregulated by the treatment with PPAR $\gamma$ agonists (Fig. 2E).

In order to confirm that the inhibition of calcification by RGZ and TZL was specifically mediated by PPAR $\gamma$, VSMCs were treated with a selective PPAR $\gamma$ antagonist, GW9662, together with RGZ or TZL. In the presence of GW9662, neither RGZ nor TZL inhibited the calcification of VSMCs (Fig. 2F). Taken together, these results indicated that activation of PPAR $\gamma$ specifically inhibited Pi-induced calcification in VSMCs.

PPAR $\gamma$ regulates the expression of Klotho in VSMCs. Since it has been reported that Klotho is a target gene of PPAR $\gamma(23)$ and that the deficiency of Klotho may cause vascular calcification $(21,22)$, it was hypothesized that activation of PPAR $\gamma$ inhibits Pi-induced calcification in VSMCs via stimulation of Klotho. To test this hypothesis, Klotho expression levels were 
A

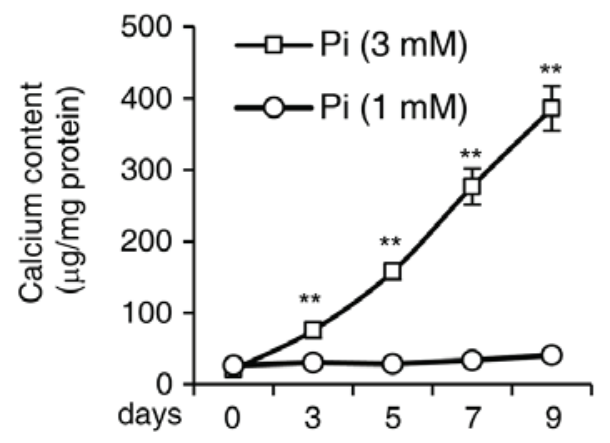

C
B
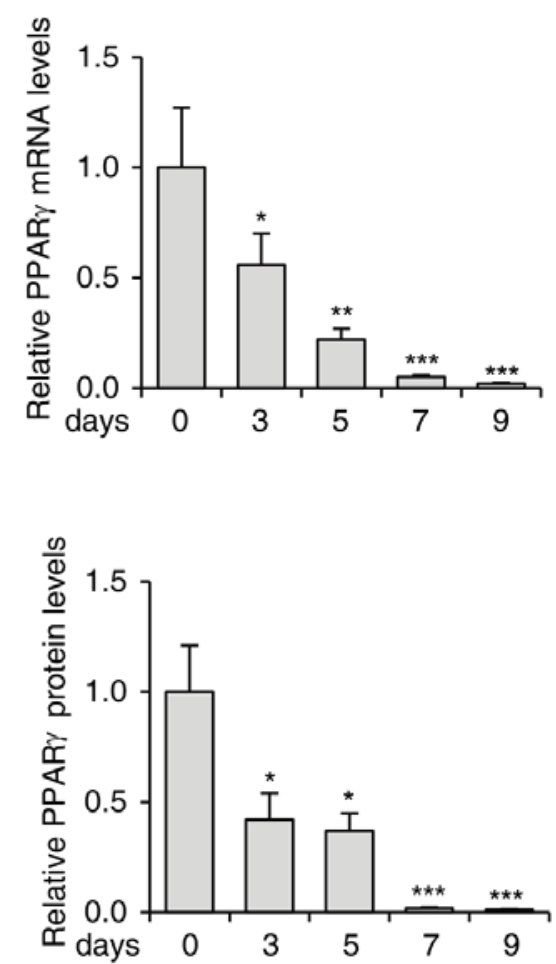

Figure 1. PPAR $\gamma$ expression is downregulated in VSMCs with Pi-induced calcification. (A) Calcium content in VSMCs treated with 1 or 3 mM Pi for $0,3,5,7$ and 9 days. (B) PPAR $\gamma$ mRNA levels in VSMCs treated with $3 \mathrm{mM}$ Pi at the indicated time points, analyzed by reverse-transcription quantitative polymerase chain reaction. (C) The protein levels of PPAR $\gamma$ in VSMCs treated with $3 \mathrm{mM}$ Pi as determined by western blot analysis. Quantified PPAR $\gamma$ protein levels are shown in the right panel. $\beta$-actin was used as the loading control. ${ }^{*} \mathrm{P}<0.05,{ }^{* *} \mathrm{P}<0.01$ and ${ }^{* * *} \mathrm{P}<0.001$ vs. cells at day 0 . Values are expressed as the mean \pm standard error of the mean of three independent experiments. VSMC, vascular smooth muscle cell; PPAR, peroxisome proliferator-activated receptor; Pi, inorganic phosphate.

first analyzed in VSMCs treated with PPAR $\gamma$ agonists RGZ and TZL during Pi-induced calcification. It was found that the expression levels of Klotho were significantly reduced in the presence of $\mathrm{Pi}$, but were upregulated by RGZ and TZL treatment (Fig. 3A). As expected, treatment with PPAR $\gamma$ inhibitor GW9662 prevented RGZ and TZL from increasing the expression levels of Klotho (Fig. 3A).

Furthermore, PPAR $\gamma$ siRNA was introduced into VSMCs to knockdown PPAR $\gamma$, and the protein levels of endogenous PPAR $\gamma$ were reduced by $\sim 80 \%$ (Fig. 3B). Knockdown of PPAR $\gamma$ further decreased Klotho expression during VSMC calcification (Fig. 3C). Importantly, when PPAR $\gamma$ expression was knocked down, RGZ and TZL could no longer upregulate the expression of Klotho, suggesting that the effects of RGZ and TZL in regulating Klotho expression were specifically mediated by PPAR $\gamma$.

PPAR r regulates VSMC calcification through activating Klotho. In order to determine whether the inhibitory effects on VSMC calcification by PPAR $\gamma$ activation were dependent on Klotho upregulation, siRNA targeting Klotho was introduced into VSMCs. With this treatment, the protein levels of endogenous Klotho were decreased by $\sim 80 \%$ (Fig. 4A). Consistently with previously reported results, Klotho knockdown led to elevated levels of Pi-induced calcification (Fig. 4B). Of note, in VSMCs with Klotho knockdown, PPAR $\gamma$ agonists were no longer able to inhibit calcification (Fig. 4B), indicating that PPAR $\gamma$ activation inhibited VSMC calcification through activating Klotho.
It has been proposed that Klotho inhibits Pi-induced VSMC calcification by inhibiting PiT-1/2 expression, thus preventing PiT-1/2-dependent Pi influx (21). In line with this theory, PPAR $\gamma$ activation induced by RGZ and TZL, as well as treatment with soluble Klotho, reduced Pi influx (Fig. 4C) and inhibited PiT-1/2 expression (Fig. 4D and E). The fact that PPAR $\gamma$ activation and Klotho reduced Pi influx and inhibited PiT-1/2 expression further suggested that the PPAR $\gamma$ activation inhibits calcification via increasing Klotho expression. Taken together, these results indicated that PPAR $\gamma$ activation ameliorated VSMC calcification through upregulation of Klotho.

\section{Discussion}

Patients with CKD have a disproportionately high occurrence of vascular calcification compared to the general population. One theory to explain this observation is the dysregulated calcium and Pi metabolism that is common in these patients. Klotho was recently found to be associated with vascular calcification $(21,22)$, possibly via its function to maintain mineral homeostasis and to regulate $\mathrm{Pi}$ influx into cells. PPAR $\gamma$ has emerged as a regulator of Klotho (23). In this context, it is important to investigate whether PPAR $\gamma$ regulates VSMC calcification through modulating the expression levels of Klotho.

The present study revealed that the expression of PPAR $\gamma$ was significantly reduced during Pi-induced VSMC calcification. This finding suggested a physiological role for PPAR $\gamma$ 
A

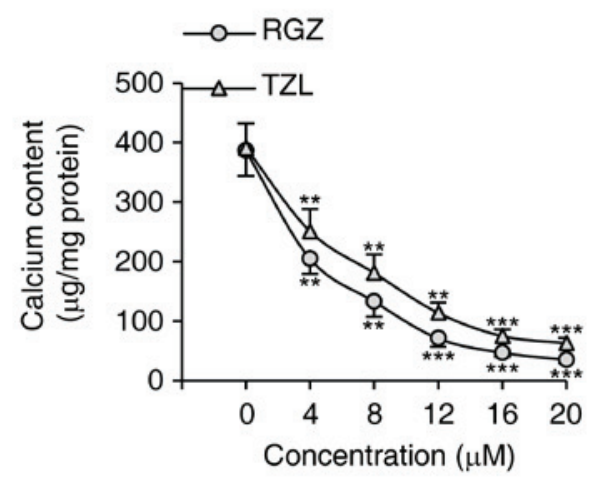

C

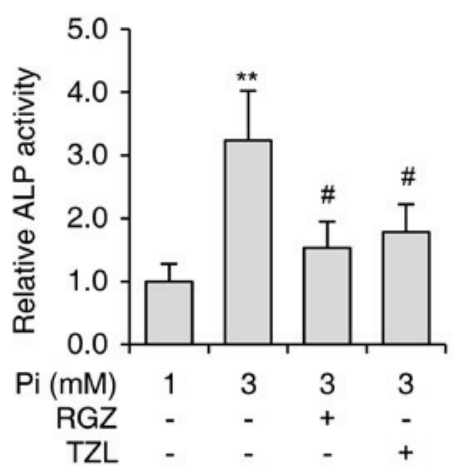

E

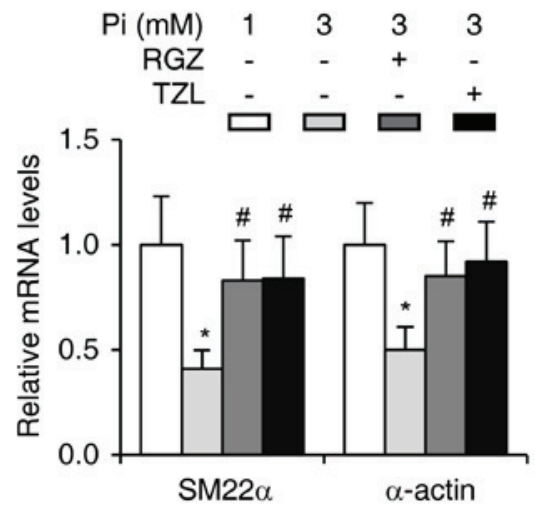

B
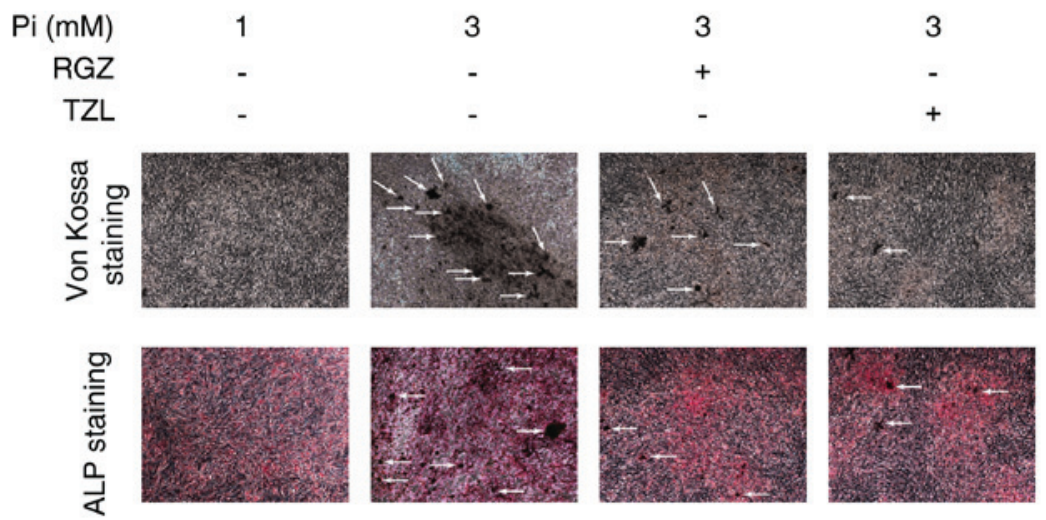

D

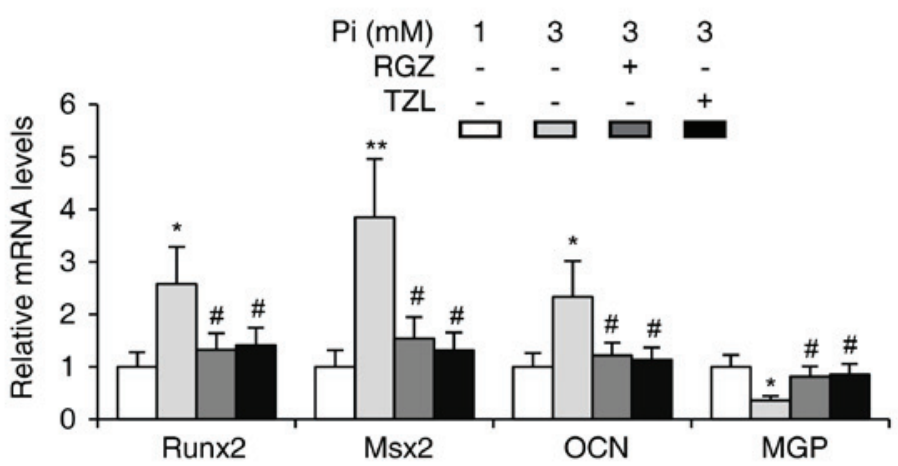

$\mathbf{F}$

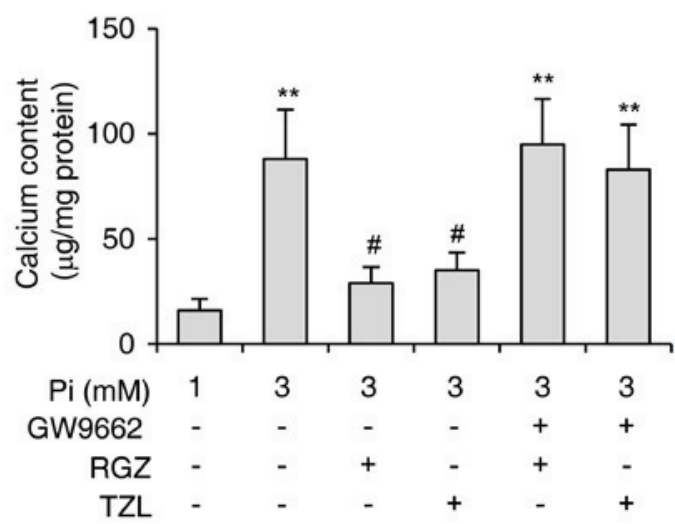

Figure 2. PPAR $\gamma$ agonists inhibit Pi-induced VSMC calcification. (A) Calcium content in VSMCs exposed to $3 \mathrm{mM}$ Pi for 9 days treated with the indicated concentrations of RGZ or TZL. ${ }^{* *} \mathrm{P}<0.01$ vs. $3 \mathrm{mM} \mathrm{Pi}$ only group, ${ }^{* * *} \mathrm{P}<0.001$ vs. $3 \mathrm{mM} \mathrm{Pi}$ only group. VSMCs were subjected to Pi-induced calcification and treated with RGZ or TZL (10 $\mu \mathrm{M}$ each), followed by (B) von Kossa (upper panel) and ALP staining (lower panel; magnification, x40; white arrows indicate calcification); (C) assessment of ALP activity; (D) determination of the mRNA expression levels of osteogenic genes Runx2, Msx2 and OCN, and calcification inhibitor MGP as well as (E) VSMC lineage markers SM22 $\alpha$ and $\alpha$-actin by reverse-transcription quantitative polymerase chain reaction analysis; and $(\mathrm{F})$ assessment of the calcium content in the absence or presence of PPAR $\gamma$ inhibitor GW9662 (10 $\mu \mathrm{M})$. Values are expressed as the mean \pm standard error of the mean of three independent experiments. $(\mathrm{C}-\mathrm{F}){ }^{*} \mathrm{P}<0.05,{ }^{* *} \mathrm{P}<0.01$ vs. cells in $1 \mathrm{mM} \mathrm{Pi}$ group. ${ }^{\#} \mathrm{P}<0.05$ vs. cells in $3 \mathrm{mM} \mathrm{Pi}$ only group. VSMC, vascular smooth muscle cell; Pi, inorganic phosphate; PPAR, peroxisome proliferator-activated receptor; TZL, thiazolidinedione; RGZ, rosiglitazone; Runx2, runt-related transcription factor 2; Msx2, Msh homeobox 2; OCN, osteocalcin; MGP, matrix gla protein; ALP, alkaline phosphatase.

in the process of VSMC calcification. In fact, PPAR $\gamma$ is a transcription factor known to act as a sensor that translates environmental stimuli into adaptive cellular responses, and has been shown to be a key repressor of osteoblastogenesis $(30,31)$. In addition, a previous study demonstrated that PPAR $\gamma$ counteracted vascular calcification by inhibiting Wnt5a signaling in atherosclerotic lesions (32). However, the mechanism of action for PPAR $\gamma$ in regulating Pi-induced vascular calcification have largely remained elusive.

The present study found that treatment with PPAR $\gamma$ agonists inhibited calcification in VSMCs in a PPAR $\gamma$-dependent manner. The calcification of VSMC was characterized not only by increased cellular calcium content, but also by phenotypic transition involving the expression of osteogenic genes. It is 
A

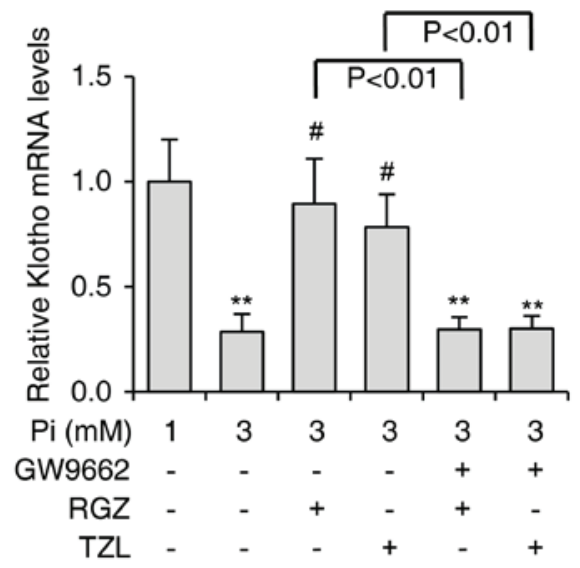

B
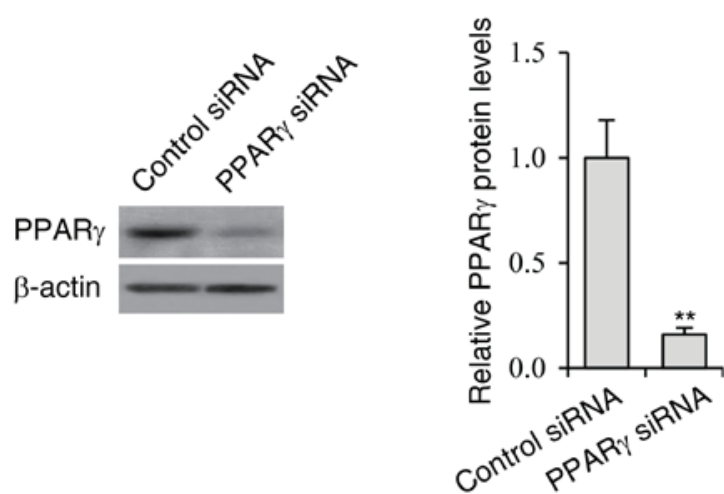

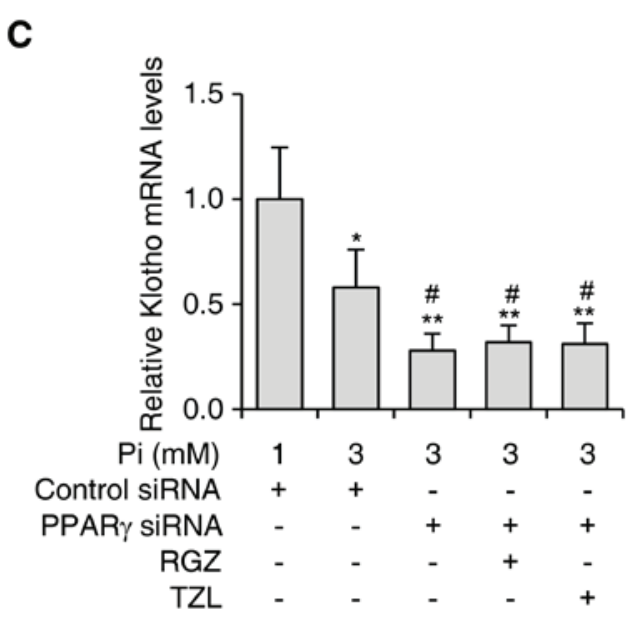

Figure 3. PPAR $\gamma$ agonists enhance Klotho expression in VSMCs. (A) VSMCs were subjected to Pi-induced calcification and treated with RGZ or TZL (10 $\mu$ M each) in the absence or presence of PPAR $\gamma$ inhibitor GW9662 $(10 \mu \mathrm{M})$. The expression levels of Klotho were determined by reverse-transcription quantitative polymerase chain reaction analysis. (B) The protein levels of PPAR $\gamma$ in VSMCs treated with siRNA targeting PPAR $\gamma$ were determined by western blot analysis. Quantified PPAR $\gamma$ protein levels are shown in the right panel. $\beta$-actin was used as the loading control. ${ }^{* *} \mathrm{P}<0.01$ vs. control siRNA-transfected cells. (C) Expression levels of Klotho in VSMCs subjected to Pi-induced calcification treated with RGZ or TZL (10 $\mu \mathrm{M})$ in the absence or presence of PPAR $\gamma$ siRNA. Values are expressed as the mean \pm standard error of the mean of three independent experiments. ${ }^{*} \mathrm{P}<0.05$, ${ }^{* *} \mathrm{P}<0.01$ vs. cells in $1 \mathrm{mM} \mathrm{Pi}$ group. ${ }^{\#} \mathrm{P}<0.05 \mathrm{vs}$. cells in 3 mM Pi only group. VSMC, vascular smooth muscle cell; Pi, inorganic phosphate; PPAR, peroxisome proliferator-activated receptor; TZL, thiazolidinedione; RGZ, rosiglitazone; siRNA, small interfering RNA.

known that VSMCs can undergo phenotypic changes (33), and that they have important roles in the initiation and progression of vascular calcification (34), which is associated with a decrease in smooth muscle lineage markers and an increase in osteogenic markers (35). The present study also found that PPAR $\gamma$ regulated the gene expression of Klotho in VSMCs. Activation of PPAR $\gamma$ led to increased expression of Klotho, while knockdown of PPAR $\gamma$ resulted in decreased levels of Klotho. These results were consistent with a previous study using kidney cell lines (23).

The most important findings of the present study were that Klotho was required for the regulation of Pi-induced vascular calcification by PPAR $\gamma$. It was demonstrated that knockdown of Klotho abolished the ability of activated PPAR $\gamma$ to inhibit calcification in VSMCs. These findings shed light on the underlying mechanisms via which PPAR $\gamma$ regulates Pi-induced vascular calcification. Of note, there are two major types of vascular calcification, which are distinguished by their location and association with atherosclerotic plaque formation.
Atherosclerotic calcification is located in the intimal layer and is associated with cellular necrosis, lipid deposition and inflammation. As lesions progress, osteogenesis becomes increasingly evident. The other type of vascular calcification is called Monckeberg sclerosis and is characterized by amorphous mineral forming along elastic lamellae of the medial layer. This type of calcification is therefore also known as medial artery calcification, and is more prevalent in patients with CKD $(6,7)$. The fact that Klotho is required for the regulation of $\mathrm{Pi}$-induced vascular calcification by PPAR $\gamma$ in medial artery calcification, which is commonly seen in CKD patients, does not exclude the possibility of further molecules mediating the regulation of vascular calcification by PPAR $\gamma$ in atherosclerotic lesions.

In conclusion, the present study showed that PPAR $\gamma$ regulates $\mathrm{Pi}$-induced calcification and Klotho expression in VSMCs. Moreover, loss of Klotho expression abolished the ability of activated PPAR $\gamma$ to inhibit VSMC calcification. To the best of our knowledge, the present study was the first to 
A

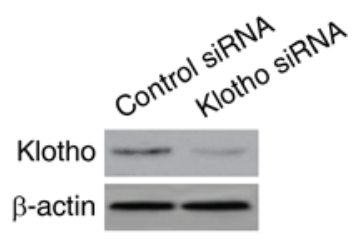

C

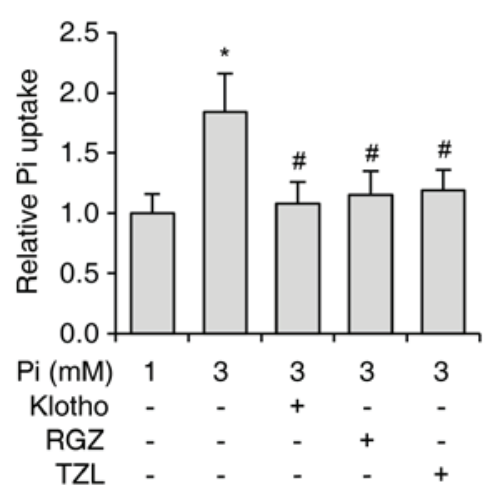

B

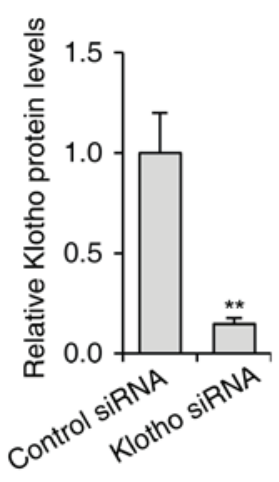

$P<0.01$

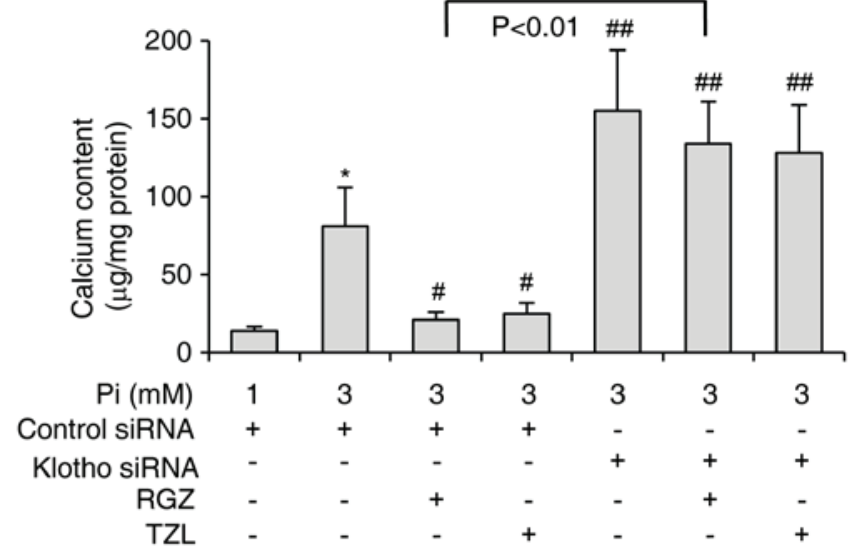

D

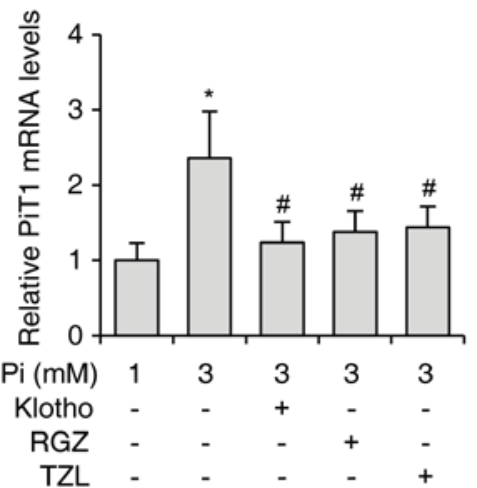

E

Figure 4. PPAR $\gamma$ regulates VSMC calcification through activating Klotho. (A) The protein levels of Klotho in VSMCs treated with siRNA targeting Klotho as determined by western blot. Quantified protein levels of Klotho are shown in the right panel. $\beta$-actin was used as the loading control. ${ }^{* *} \mathrm{P}<0.01$ vs. control siRNA-transfected cells. (B) Calcium content in VSMCs subjected to Pi-induced calcification treated with RGZ or TZL (10 $\mu$ M each), in the absence or presence of Klotho siRNA. (C) Pi uptake and (D) PiT-1 mRNA levels and (E) PiT-2 mRNA levels as determined by reverse-transcription quantitative polymerase chain reaction analysis in VSMCs subjected to Pi-induced calcification and treated with RGZ (10 $\mu \mathrm{M})$, TZL (10 $\mu \mathrm{M})$ or soluble Klotho (0.4 nM). Values are expressed as the mean \pm standard error of the mean of three independent experiments. ${ }^{* *} \mathrm{P}<0.05 \mathrm{vs}$. control siRNA-transfected cells treated with $1 \mathrm{mM}$ Pi. ${ }^{\#} \mathrm{P}<0.05,{ }^{\# \#} \mathrm{P}<0.01$ vs. control siRNA-transfected cells treated with $3 \mathrm{mM}$ Pi. VSMC, vascular smooth muscle cell; Pi, inorganic phosphate; TZL, thiazolidinedione; RGZ, rosiglitazone; siRNA, small interfering RNA; PiT, Pi transporter.

demonstrate that PPAR $\gamma$ regulates VSMC calcification through activating Klotho.

\section{Acknowledgements}

This work was supported by the Specialized Research Fund for the Doctoral Program of Higher Education of China (grant no. 20122307110011), Science Projects of Heilongjiang Health Department (grant no. 2013024); and Key Projects of Natural Science Foundation of Heilongjiang Province (grant no. ZD201014).

\section{References}

1. Foley RN, Parfrey PS and Sarnak MJ: Clinical epidemiology of cardiovascular disease in chronic renal disease. Am J Kidney Dis 32 (5 Suppl 3): S112-S119, 1998.

2. Goodman WG, Goldin J, Kuizon BD, Yoon C, Gales B, Sider D, Wang Y, Chung J, Emerick A, Greaser L, et al: Coronary-artery calcification in young adults with end-stage renal disease who are undergoing dialysis. N Engl J Med 342: $1478-1483,2000$.
3. McCullough PA, Sandberg KR, Dumler F and Yanez JE: Determinants of coronary vascular calcification in patients with chronic kidney disease and end-stage renal disease: A systematic review. J Nephrol 17: 205-215, 2004.

4. London GM, Guérin AP, Marchais SJ, Métivier F, Pannier B and Adda $\mathrm{H}$ : Arterial media calcification in end-stage renal disease: Impact on all-cause and cardiovascular mortality. Nephrol Dial Transplant 18: 1731-1740, 2003.

5. Shroff RC, McNair R, Figg N, Skepper JN, Schurgers L, Gupta A, Hiorns M, Donald AE, Deanfield J, Rees L and Shanahan CM: Dialysis accelerates medial vascular calcification in part by triggering smooth muscle cell apoptosis. Circulation 118: 1748-1757, 2008 .

6. Goodman WG, London G, Amann K, Block GA, Giachelli C, Hruska KA, Ketteler M, Levin A, Massy Z, McCarron DA, et al: Vascular calcification in chronic kidney disease. Am J Kidney Dis 43: 572-579, 2004.

7. Shroff RC and Shanahan CM: The vascular biology of calcification. Semin Dial 20: 103-109, 2007.

8. Block GA, Klassen PS, Lazarus JM, Ofsthun N, Lowrie EG and Chertow GM: Mineral metabolism, mortality, and morbidity in maintenance hemodialysis. J Am Soc Nephrol 15: 2208-2218, 2004.

9. Cozzolino M, Dusso AS and Slatopolsky E: Role of calcium-phosphate product and bone-associated proteins on vascular calcification in renal failure. J Am Soc Nephrol 12: 2511-2516, 2001. 
10. Ganesh SK, Stack AG, Levin NW, Hulbert-Shearon T and Port FK: Association of elevated serum PO(4), Ca x PO(4) product, and parathyroid hormone with cardiac mortality risk in chronic hemodialysis patients. J Am Soc Nephrol 12: 2131-2138, 2001.

11. Boström K, Watson KE, Horn S, Wortham C, Herman IM and Demer LL: Bone morphogenetic protein expression in human atherosclerotic lesions. J Clin Invest 91: 1800-1809, 1993.

12. Iimura T, Oida S, Takeda K, Maruoka Y and Sasaki S: Changes in homeobox-containing gene expression during ectopic bone formation induced by bone morphogenetic protein. Biochem Biophys Res Commun 201: 980-987, 1994.

13. Shanahan CM, Cary NR, Salisbury JR, Proudfoot D, Weissberg PL and Edmonds ME: Medial localization of mineralization-regulating proteins in association with Mönckeberg's sclerosis: Evidence for smooth muscle cell-mediated vascular calcification. Circulation 100: 2168-2176, 1999.

14. Tyson KL, Reynolds JL, McNair R, Zhang Q, Weissberg PL and Shanahan CM: Osteo/chondrocytic transcription factors and their target genes exhibit distinct patterns of expression in human arterial calcification. Arterioscler Thromb Vasc Biol 23 489-494, 2003

15. Block GA, Hulbert-Shearon TE, Levin NW and Port FK: Association of serum phosphorus and calcium $\mathrm{x}$ phosphate product with mortality risk in chronic hemodialysis patients: A national study. Am J Kidney Dis 31: 607-617, 1998.

16. Jono S, McKee MD, Murry CE, Shioi A, Nishizawa Y, Mori K, Morii $\mathrm{H}$ and Giachelli CM: Phosphate regulation of vascular smooth muscle cell calcification. Circ Res 87: E10-E17, 2000.

17. Li X, Yang HY and Giachelli CM: Role of the sodium-dependent phosphate cotransporter, Pit-1, in vascular smooth muscle cell calcification. Circ Res 98: 905-912, 2006.

18. Lomashvili K, Garg P and O'Neill WC: Chemical and hormonal determinants of vascular calcification in vitro. Kidney Int 69: 1464-1470, 2006.

19. Yang H, Curinga $\mathrm{G}$ and Giachelli CM: Elevated extracellular calcium levels induce smooth muscle cell matrix mineralization in vitro. Kidney Int 66: 2293-2299, 2004.

20. Kuro-o M: Klotho. Pflugers Arch 459: 333-343, 2010.

21. Hu MC, Shi M, Zhang J, Quiñones H, Griffith C, Kuro-o M and Moe OW: Klotho deficiency causes vascular calcification in chronic kidney disease. J Am Soc Nephrol 22: 124-136, 2011.

22. Lim K, Lu TS, Molostvov G, Lee C, Lam FT, Zehnder D and Hsiao LL: Vascular Klotho deficiency potentiates the development of human artery calcification and mediates resistance to fibroblast growth factor 23. Circulation 125: 2243-2255, 2012.

23. Zhang H, Li Y, Fan Y, Wu J, Zhao B, Guan Y, Chien S and Wang N: Klotho is a target gene of PPAR-gamma. Kidney Int 74 732-739, 2008
24. Zhou YB, Zhang J, Peng DQ, Chang JR, Cai Y, Yu YR, Jia MZ, Wu W, Guan YF, Tang CS and Qi YF: Peroxisome proliferator-activated receptor $\mathrm{g}$ ligands retard cultured vascular smooth muscle cells calcification induced by high glucose. Cell Biochem Biophys 66: 421-429, 2013.

25. Ross R: The smooth muscle cell. II. Growth of smooth muscle in culture and formation of elastic fibers. J Cell Biol 50: 172-186, 1971.

26. Shioi A, Nishizawa Y, Jono S, Koyama H, Hosoi M and Morii H: Beta-glycerophosphate accelerates calcification in cultured bovine vascular smooth muscle cells. Arterioscler Thromb Vasc Biol 15: 2003-2009, 1995.

27. Livak KJ and Schmittgen TD: Analysis of relative gene expression data using real-time quantitative PCR and the 2(-Delta Delta C(T)) Method. Methods 25: 402-408, 2001.

28. Rungby J, Kassem M, Eriksen EF and Danscher G: The von Kossa reaction for calcium deposits: Silver lactate staining increases sensitivity and reduces background. Histochem J 25: 446-451, 1993.

29. Hui M, Li SQ, Holmyard D and Cheng P: Stable transfection of nonosteogenic cell lines with tissue nonspecific alkaline phosphatase enhances mineral deposition both in the presence and absence of beta-glycerophosphate: Possible role for alkaline phosphatase in pathological mineralization. Calcif Tissue Int 60: 467-472, 1997.

30. Wan Y: PPARgamma in bone homeostasis. Trends Endocrinol Metab 21: 722-728, 2010.

31. Wan Y, Chong LW and Evans RM: PPAR-gamma regulates osteoclastogenesis in mice. Nat Med 13: 1496-1503, 2007.

32. Woldt E, Terrand J, Mlih M, Matz RL, Bruban V, Coudane F, Foppolo S, El Asmar Z, Chollet ME, Ninio E, et al: The nuclear hormone receptor PPARg counteracts vascular calcification by inhibiting Wnt5a signalling in vascular smooth muscle cells. Nat Commun 3: 1077, 2012.

33. Iyemere VP, Proudfoot D, Weissberg PL and Shanahan CM: Vascular smooth muscle cell phenotypic plasticity and the regulation of vascular calcification. J Intern Med 260: 192-210, 2006.

34. Speer MY, Yang HY, Brabb T, Leaf E, Look A, Lin WL, Frutkin A, Dichek D and Giachelli CM: Smooth muscle cells give rise to osteochondrogenic precursors and chondrocytes in calcifying arteries. Circ Res 104: 733-741, 2009.

35. Steitz SA, Speer MY, Curinga G, Yang HY, Haynes P, Aebersold R, Schinke T, Karsenty G and Giachelli CM: Smooth muscle cell phenotypic transition associated with calcification: Upregulation of Cbfa1 and downregulation of smooth muscle lineage markers. Circ Res 89: 1147-1154, 2001. 